\title{
Corela
}

Cognition, représentation, langage

HS-19 | 2016

Le point de vue pris au mot

\section{À la recherche des points de vue dans la langue}

\author{
Marta Tordesillas
}

\section{(2) OpenEdition}

\section{Journals}

Édition électronique

URL : http://journals.openedition.org/corela/4270

DOI : $10.4000 /$ corela.4270

ISSN : $1638-573 X$

Éditeur

Cercle linguistique du Centre et de l'Ouest - CerLICO

\section{Référence électronique}

Marta Tordesillas, « A la recherche des points de vue dans la langue », Corela [En ligne], HS-19 | 2016, mis en ligne le 08 juin 2016, consulté le 02 mai 2019. URL : http://journals.openedition.org/ corela/4270 ; DOI : 10.4000/corela.4270

Ce document a été généré automatiquement le 2 mai 2019.

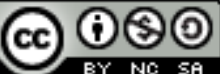

Corela - cognition, représentation, langage est mis à disposition selon les termes de la licence Creative Commons Attribution - Pas d'Utilisation Commerciale - Partage dans les Mêmes Conditions 4.0 International. 


\title{
À la recherche des points de vue dans la langue
}

\author{
Marta Tordesillas
}

\section{Introduction}

1 Depuis des siècles, les recherches sur le langage et la langue ne cessent de se développer. Maintes sont les réflexions qui, d'abord, dans le cadre de la philosophie, de la rhétorique ou de la logique, puis de la psychologie, sociologie, anthropologie et, enfin, de la sémiotique et de la linguistique, réfléchissent à la conception du langage et de la langue en essayant de rendre compte de leurs configurations et de leurs fonctions. Au long du XX e siècle, plusieurs aspects clés surgissent d'une façon particulièrement intéressante, notamment ceux qui sont en relation avec la composante dite sémantique ou pragmatique de la langue, mise à l'écart de la Science pendant des siècles. Les réflexions à ce sujet de Bréal, Bally, Bakhtine ou Benveniste, entre autres, restent décisives et mettent l'accent sur de nombreux problèmes de linguistique générale concernant le sens, questions qui, de nos jours, constituent nos principaux défis. Ainsi, ils se posent des questions sur les relations entre le langage et la vie, le langage et la société, le langage naturel et le langage formel, le langage et la langue, la langue et le discours, la typologie et les genres du discours entre autres problématiques. Ils s'interrogent de même sur l'évolution du langage et de la linguistique générale, sur le psychologique, l'anthropologique, le biologique, le neurologique, ... le linguistique et le culturel. Ils s'interpellent sur la pensée, le symbole, le signe et l'objet, le sujet et le monde, sur les composantes linguistiques et les niveaux d'analyse intralinguistique et, encore, sur le lien entre le langage et l'être humain, entre l'insconscient et l'empreinte en langue ou entre la subjectivité et la société, en y attachant une considération particulière aux langues réelles en tant qu'organismes empiriques demeurant le seul accès possible pour l'appréhension des fondements du langage. C'est dans ce cadre qu'un premier problème se situe au centre de l'intérêt scientifique en général et des sciences du langage en particulier : la relation de l'être humain avec le langage et la langue, puis du langage et de la langue avec 
l'être humain. Une deuxième situation scientifique est tout aussi très présente, il s'agit de l'essence même, objective / subjective, de la langue.

2 Il est clair que ces deux questions scientifiques vont développer une recherche très poussée qui prend sens non seulement pour connaître la conformation et la configuration du langage et de la langue, mais aussi pour s'approcher de la connaissance de l'être humain et des relations et interactions discursives socioculturelles, et, puis, pour assurer le développement technologique fondé sur la langue. Il importe justement de s'interroger sur la place qui, encore de nos jours, est attribuée au langage dans la définition de l'être humain, dès le moment où, en effet, le langage est conçu comme une faculté dont les manifestations sont des instruments naturels pour s'inscrire dans la / les société / s et dans le / s monde / s, mais aussi, en tant qu'hypothèse plus récente, il peut être considéré comme constructeur et modalisateur de société $/ \mathrm{s}$ et de monde $/ \mathrm{s}^{2}$. Cette idée est à mettre en relation avec la réflexion liée à l'unicité de la langue, à la pensée unique, au monde unique, au vrai... et, pourquoi pas, à la force économique unique ! ${ }^{3}$ Les dites circonstances scientifiques se posent donc dans le cadre de la linguistique générale et, bien qu'étant une problématique présente auparavant, elle ne devient vraiment essentielle qu'à partir de 1966. C'est alors où les chercheurs vont s'intéresser, de façon collective, à un sujet qui nous semble particulièrement pertinent, à la composante sémantique, au principe de subjectivité et au concept d'énonciation et d'argumentation dans la langue. Lesdits concepts vont se situer à la base de l'essor inarrêtable des recherches en sémantique et en pragmatique et vont devenir une réponse aux faits de langage, de langue et de discours, ce qui favorisera une description plus précise de la configuration de la langue et de ses réalisations. D'ailleurs, ils vont devenir la base du développement technologique des communications. Les exemples les plus clairs et les propositions théoriques les plus évoluées surgissent notamment dans la seconde moitié du XXe siècle et trouvent leur plus grand echo au sein de deux théories : il s'agit des théories, dans les différentes étapes de son développement, de L'Argumentation dans la langue (1983) et de la théorie de la polyphonie énonciative (1984). Les recherches menées dans ce cadre-ci vont donner lieu, à Paris, au développement d'une nouvelle École de linguistique, l'École argumentativiste de Paris.

\section{L'École argumentativiste de Paris}

3 L'une des principales Écoles de linguistique générale de la fin du XX $\mathrm{XX}^{\mathrm{e}}$ siècle, c'est, en effet, l'École argumentativiste de Paris, insuffisamment reconnue, à notre avis, dans le cadre des Science du langage. Elle trouve son origine dans les réflexions philosophiques séculaires sur la subjectivité, dont certaines vont être identifiées, reprises, synthétisées, redéfinies, amplifiées et enrichies. Elles constituent ainsi les fondements pour le développement d'un nouveau cadre philosophique, scientifique, linguistique, théorique et méthodologique, concevant alors de nouveaux objets scientifiques, de nouveaux observables, de nouveaux concepts. La base scientifique de ce cadre formule l'inscription de l'argumentation et de l'énonciation, de façon intrinsèque ${ }^{4}$, dans la signification. Le philosophe et linguiste qui donne lieu, en tant que tel, à ce cadre théorique est Oswald Ducrot, qui, en collaboration avec le mathématicien et linguiste Jean-Claude Anscombre, propose une conception argumentative de la langue. Il faut dire que Ducrot, dès 1970, pose une conception pionnière sur la langue, qui formule des nouveautés conceptuelles, descriptives et méthodologiques, essentielles sur la langue et innovantes pour la 
linguistique générale et les sciences du langage. En voici certains concepts dont va s'occuper la linguistique ducrotienne: le structuralisme, la signification, le sens, le discours, le dire, le dit, l'implicite, l'argumentation, l'énonciation, les présupposés, les posés, les mots du discours, les échelles argumentatives, la gradualité, les variables argumentatives, l'argument, la conclusion, l'orientation, les topoi, les formes topiques, etc. Juste déjà la conceptualisation montre en elle-même l'originalité de la théorie formulée sur la langue. Bien que cette conception de l'argumentation dans la langue ait subi différentes étapes et développements, il est intéressant de citer certaines publications qui se trouvent à l'origine, du fait qu'elles mettent l'accent sur des sujets, des observables et des concepts, exclus, à cette époque-là, de toute visée scientifique et linguistique. Elles constituent d'ailleurs une révolution de la pensée linguistique. Certains ouvrages ont rassemblé, synthétisé et explicité lesdits fondements théoriques et en font la preuve des nouveautés scientifiques: Ducrot $(1973)^{5}$, Anscombre et Ducrot (1976) et Ducrot (1980), Les échelles argumentatives; Ducrot et alii (1980), Les mots du discours; Anscombre et Ducrot (1983), L'Argumentation dans la langue; Ducrot (1984), Le dire et le dit, où l'auteur expose, dans le chapitre 8 , l'esquisse pour une théorie polyphonique de l'énonciation.

\subsection{Les écrits fondateurs}

4 Les auteurs des ouvrages cités ci-dessus présentent à la communauté scientifique la conception théorique développée qui, tout en reprenant certains concepts classiques du cadre de la rhétorique, les définissent, les reformulent, les enrichissent et, surtout, ils les inscrivent dans la langue, de telle sorte que la proposition théorique constitue une nouveauté. Leurs écrits, qui souvent réunissent des études et des articles préalablement publiés par les auteurs, représentent le point de repère de la formulation théorique qu'ils proposent.

5 Ainsi, un des premiers livres, intitulé Les échelles argumentatives (1980) constitue un fondement tout à fait original pour la conception de la langue et, cela, au moins pour deux raisons. La première est l'hypothèse selon laquelle l'argumentation est dans la langue, et non seulement dans le discours, et comporte un principe de stratégie, de visée, d'orientation, d'enchaînement associés à la langue elle-même, à la configuration du sens, ce qui permet de mettre en question le caractère exclusivement informatif de la langue. La deuxième, c'est l'idée même d'échelle qui, liée à la langue, comporte déjà, dans la signification même, une composante dynamique et graduelle qui met en question le caractère objectif, passif et fixe de la langue et, par extension du signe, tel qu'il fut conçu par Saussure. Ducrot expliquera :

Le thème central de la théorie argumentative est que le sens d'un énoncé contient une allusion à son éventuelle continuation : il lui est essentiel d'appeler tel ou tel type de suite, de prétendre orienter le discours ultérieur dans telle direction. S'il est argumentatif, ce n'est pas seulement par ce qu'il dit sur le monde, mais par ce qu'il est, si on le considère en lui-même. Certes, on ne saurait prévoir ce qui va effectivement lui faire suite [...] Mais il a une suite "prétendue ", celle qu'il donne comme sa raison d'être, et celle-ci est autant en lui que hors de lui. Par là, la théorie argumentative se relie à la théorie générale que j'ai appelée «structuralisme du discours idéal» selon laquelle une entité linguistique tire toute sa réalité du discours où elle prend place. (Ducrot 1980 : 11) 
Puis, louvrage Les mots du discours (1980) cherche à montrer que tous les mots de la langue ont un sens....argumentatif, une signification, et aussi ceux qui, au long des siècles, ont été considérés par de nombreux grammairiens et de nombreux linguistes comme des mots vides, les conjonctions. La recherche sur le sens et, notamment, l'hypothèse d'un sens argumentatif permet de constater que les mots dits du discours, connecteurs et opérateurs, comportent en eux-mêmes des instructions qui, d'ailleurs, sont clés pour l'appréhension du sens dans son niveau horizontal et vertical. L'idée d'un livre sur les connecteurs et les opérateurs met déjà en évidence l'importance de ces éléments de la langue et l'attention sicentifique portée à ce moment. Ducrot dira :

Comprendre un discours c'est toujours imaginer des stratégies de ce genre. Et nous pensons que la linguistique peut y aider - dans la mesure où elle donne aux mots, donc aux phrases, des significations qui obligent, pour se laisser transformer en sens, à reconstituer les débats dont le discours est le lieu. (Ducrot et alii 1980 : 56)

Et encore, le livre L'Argumentation dans la langue (1983), issu d'une tradition structuraliste, qui a pour objectif d'aboutir à une description sémantique des énoncés, ouvre une perspective dans laquelle les auteurs rendront compte, plus tard, du lexique de la langue, en affirmant que le sens est lié à des variables argumentatives, plus précisément que des contraintes intrinsèques sur le sens opèrent entre les variables argumentatives d'argument et de conclusion. La valeur sémantique d'un énoncé est constituée par l'allusion à la possibilité d'un autre énoncé. Cette formulation s'éloigne à nouveau du principe selon lequel la langue informe, pour en poser que c'est l'argumentation le vecteur sémantique directeur $\mathrm{du}$ sens. Le caractère informatif de la langue est alors remplacé par le principe selon lequel la langue argumente.

C'est ainsi que Ducrot élabore avec Anscombre une théorie de l'argumentation dans la langue qui consiste à saisir le déploiement de l'argumentation, les potentialités linguistiques au niveau de la langue elle-même. L'idée maîtresse est que la description de la langue n'a pas comme but principal la représentation du monde, mais l'argumentation. La langue constitue un système où l'on trouve des arguments, des enchaînements, des conclusions, dont la structure est logée dans la signification de la langue. Dans l'avantpropos du livre les auteurs indiquent :

[...] notre réponse repose sur une idée centrale, que nous voudrions simplement rendre plus explicite : le sens d'un énoncé comporte, en tant que partie intégrante et constitutive, cette forme d'influencer que l'on appelle la force argumentative. Signifier, pour un énoncé, c'est orienter. De sorte que la langue, dans la mesure où elle contribue en première place à déterminer le sens des énoncés, est un des lieux privilégiés où s'élabore l'argumentation. (Anscombre et Ducrot $1983: 1$ )

9 Ducrot et Anscombre établissent une distinction entre signification et sens, entre la signification de la phrase et le sens de l'énoncé. La différence, signale Ducrot, est de nature. La signification ne se retrouve pas dans le sens à titre de partie : elle est, pour l'essentiel..., constituée de directives, ou encore d'instructions, de consignes, pour instruire le sens des énoncés.

10 Il est à signaler que la théorie de l'argumentation dans la langue a subi plusieurs étapes vers une radicalisation des affirmations des hypothèses. C'est dans ce sens où s'établit une deuxième étape qui est illustrée par la théorie des topoi qui se fonde sur le concept de topos (et de formes topiques), vu, au départ comme un garant, assurant l'articulation entre l'énoncé-argument et l'énoncé-conclusion, puis l'enchaînement des variables. Le topos est considéré comme inscrit dans la signification même des énoncés, des lexèmes, de façon à formuler qu'utiliser un mot c'est convoquer des topoi, faisceaux de topoi.

Corela, HS-19 | 2016 
11 Lié à l'argumentation, Ducrot développe un concept capital pour les sciences du langage, c'est la polyphonie énonciative. Dans ce cadre-là, les premiers travaux sont réunis dans l'ouvrage Dire et ne pas dire. Principes de sémantique ([1972] 1980), où l'auteur propose des concepts tels que l'implicite, la présupposition, la supposition, avec des questions liées aux indications qu'apportent l'acte d'énonciation en mettant en relief la distinction entre ce dont il semble qu'« on » entend explicitement informer l'auditeur, mais aussi et surtout ce que le sens de l'énoncé présente comme un acquis indiscutable dont on fait le cadre du dialogue. Puis, il resterait aussi à considérer ce que l'énoncé laisse à l'auditeur le soin de deviner, sans prendre la responsabilité de l'avoir dit. D'après l'auteur d'ailleurs une sémantique qui s'en tiendrait au niveau de l'explicite serait totalement artificielle : elle rendrait incompréhensible le discours et serait suceptible de défigurer la langue ellemême; c'est en effet, selon le Ducrot de l'époque, un trait inhérent à la langue, et l'un de ses traits les plus constants et les plus fondamentaux, que de permettre aux interlocuteurs d'instituer entre eux un réseau de rapports implicites.

En ce qui concerne l'énonciation, il est à préciser cependant que le livre clef c'est Le dire et le dit (1984), notamment le chapitre VIII, Esquisse d'une théorie polyphonique de l'énonciation, où l'auteur détruit l'axiome classique de l'unicité du sujet parlant et, avec cela, l'idée de relation directe entre la pensée et la langue, pour formuler la polyphonie comme la caractéristique primaire du sens. Ducrot formule que «tout énoncé comporte une qualification de son énonciation, qualification qui constitue le sens de l'énoncé. [...] Pour cela, il faut décrire systématiquement les images de l'énonciation qui sont véhiculées à travers l'énoncé» (Ducrot 1984: 174). Le sens de l'énoncé est considéré comme une cristallisation de voix virtuelles, il est composé de figures discursives, telles que le locuteur (en tant que tel et en tant qu'être du monde) et les énonciateurs.

13 La théorie de la polyphonie énonciative permet de remplacer l'analyse horizontale du sens par une analyse verticale. L'idée de base est que le sens de l'énoncé est constitué par la superposition de plusieurs discours et / ou voix élémentaires, dont les responsables prétendus, que Ducrot appelle énonciateurs, peuvent être tout à fait différents du responsable que l'énoncé s'attribue à lui-même, et que Ducrot appelle locuteur. La signification de la phrase indique les différents énonciateurs mis en jeu lorsqu'on aura à analyser l'énoncé concret. De plus, le sens de l'énoncé montre certaines contraintes que l'on devra respecter si l'on cherche à assimiler ces énonciateurs à des êtres déterminés, ce qui n'est pas nécessairement prévu par la théorie elle-même.

\subsection{Développements de l'École}

14 C'est à la lueur des considérations, formulations, hypothèses et concepts conçus dans les théories abordées dans les ouvrages précédents que se développe un courant de recherche que l'on peut appeller École argumantitiviste de Paris. Les chercheurs ${ }^{6}$ que l'on peut, à l'origine, identifier à cette école sont nombreux bien que certains soient plus marqués parmi d'autres qui, par leur formulation théorique, ont contribué à la réflexion ou au progrès de cette perspective théorique et même qui ont formulé une théorie linguistique à l'issu des bases de pensée et des fondements théoriques de l'argumentation et de l'énonciation dans la langue. Nous pouvons de même associer à cette École tout aussi de nombreux disciples ${ }^{7}$ de Ducrot, dont nous citerons ceux qui ont, dans une certaine mesure, favorisé le développement, moyennant des études de recherche, de nouveaux concepts théoriques et méthodologiques ou un cadre théorique plus large 
visant la description complète de la langue ${ }^{8}$. C'est dans ce sens où l'on peut faire appel aux théories les plus saillantes, ainsi Ducrot et Anscombre, la Théorie de l'Argumentation dans la langue (TAL); Ducrot, la Théorie polyphonique de l'énonciation; Ducrot et Anscombre, la Théorie des Topoi; Ducrot et Carel, la Théorie des Blocs sémantiques (TBS) ; Anscombre, la Théorie des stéréotypes; Galatanu, la Théorie Sémantique des possibles argumentatifs (SPA); Raccah, la Sémantique des points de vue (SPV); Tordesillas, la Théorie de la Sémantique énonciative et argumentative (SAE); et bien d'autres théories, plus ou moins axées sur l'argumentation et / ou sur l'énonciation. Plusieurs des cadres auxquels nous venons de faire appel partagent un certain lien et, cela, à travers différents fondements qui constituent la langue. Un des concepts partagés auquel nous nous intéresserons dans les pages qui suivent et qui est présent dans la plupart desdites théories sur l'énonciation, c'est le concept de point de vue. Il est vrai est que sa définition est susceptible de différer selon le caractère qu'on lui attribue dans chacune des théories ou bien selon le rôle qu'il joue dans la langue et dans la description linguistique. En fait, il est à signaler que, depuis l'an 2000, c'est un concept qui occupe une place centrale dans de nombreuses recherches, étant la Théorie de la Polyphonie énonciative de Ducrot à l'origine des différentes perspectives et la Sémantique des points de vue de Raccah l'une des plus spécifiques dans ce sens.

\section{L'histoire du concept de langue}

Le cadre théorique dont nous nous occupons au long de ces pages, nous tenons à y insister 9 , tout en étant scientifiquement reconnu, continue cependant à se heurter à des axiomes historiques qui se maintiennent actifs depuis des siècles et constituent de véritables freins au progrès scientifique des études sur le sens. Actuellement, les raisons qui sont encore susceptibles de constituer des entraves auxquelles se heurte la recherche en Sciences du langage sont, entre autres, les suivantes :

- le poids de l'histoire philosophique sur la pensée scientifique ;

- le choix de certains principes philosophiques restrictifs au détriment d'une référence scientifique large, plurielle et partagée ;

- la méfiance de la Science à l'égard de nouvelles conceptions, différentes, sensibles;

- le coup de l'effort d'une révision des bases scientifiques, théoriques et méthodologiques;

- la fixité des savoirs et de l'éducation ${ }^{10}$;

- le modèle technologique initialement produit qui a reproduit la tangibilité des objets scientifiques;

- le pouvoir social et économique de la langue ;

- la connaissance de la langue et le développement de l'esprit critique et de la liberté.

Lesdites circonstances se traduisent dans des contraintes spécifiques sur la réflexion sur langue et sur sa conception, notamment sémantique, qui, tout en étant très restrictives au long des siècles, deviennent, de nos jours, inaceptables car elles risquent de s'imposer définitivement dans le développement du monde humain, du monde numérique et de construire, malheureusement, à nouveau, des univers discursifs et des mondes humains et techniques de pouvoir, d'individualité, de déséquilibre et d'inégalité. Nous souhaitons donc expliciter la situation, pour provoquer une prise de conscience sur ces restrictions sur la Science et sur le monde, qui risquent constamment de bousculer à nouveau les anciennes et les nouvelles propositions et d'insister sur le besoin de les dépasser 
clairement et d'inclure, définitivement, de nouveaux paradigmes scientifiques auxquels nous avons fait appel pour la conception scientifique du sens.

La pensée et la recherche historiques sur le langage et la langue montrent d'ailleurs qu'il existe plusieurs contraintes et de nombreux intérêts qui ont empêché l'apparition et l'évolution de la sémantique au long des siècles. Dans ce qui suit, nous allons les identifier pour les dépasser, ainsi :

- La pensée philosophique et son lien à des considérations idéologiques, politiques, religieuses, économiques ;

- La philosophie, le Monde et le Vrai qui determinent la conception du langage du visible ;

- La formulation d'un sujet parlant unique où la langue est le reflet de la pensée ;

- La considération de la linguistique comme un art avant même de la concevoir comme une science ${ }^{11}$;

- La conception logique, informative, représentative, référentielle et passive de la communication ;

- Le cadre méthodologique contraignant le traitement de la langue, qui observe la forme, ainsi la morphologie, la phonologie et la syntaxe;

- la mise à l'écart des études sur le sens, notamment connotatif, métaphorique et subjectif, pendant des siècles;

- Le caractère d'inappréhensibilité attribué au sens en tant qu'observables directs et objet scientifique ;

- La description de la langue avec un métalangage dont les termes proviennent de la langue en elle même;

- La relation directe de la langue avec la société, la condition humaine et le monde et, même aussi, avec la technologie ;

- L'opinion de l'être humain qui croit que par le fait de parler il sait parler pertinemment, même s'il a du mal à dire ce qu'il veut dire et, très souvent, il reformule son discours sans trop de réflexion;

£. L'interprétation des discours, plutôt que la compréhension, dans un sens polémique devenant des lieux de conflits, bien qu'il serait bon qu'ils soient plutôt faits pour la rencontre ;

- L'utilisation que fait l'être humain de la langue partout et pour tout faire au point de régir les relations socioéconomiques et technologiques.

L'entrelacement de toutes ses variables a conduit, jusqu'aux années $60 \mathrm{du} \mathrm{XX}^{\mathrm{e}}$ siècle, au développement d'une linguistique saussurienne et d'un système de la communication que Raccah qualifie comme la métaphore de la transmission (ou du tuyau), qui correspond au schéma du circuit de la parole et / ou, par extension, au schéma de la communication / information de Saussure ([1916] 1972: 27). Il est alors à signaler que la conception saussurienne, tel qu'il est prouvé d'ailleurs au long du $\mathrm{XX}^{\mathrm{e}}$ siècle, ne peut pas servir de base à une étude scientifique des contraintes que les langues imposent à la construction du sens. Par rapport à cette conception saussurienne informative de la communication et de la langue, de nombreux chercheurs, philosophes, littéraires, linguistes, mathématiciens, font remarquer, à partir de la seconde moitié du $\mathrm{XX}^{\mathrm{e}}$ siècle, à la communauté scientifique, la problématique qu'une conception de ce type entraîne pour la conceptualisation sémantique de la langue et mettent en relief de nombreux problèmes qui en dérivent ${ }^{12} \mathrm{du}$ moment où le sens reste à l'écart de la description de la langue. Une réaction scientifique reste donc à faire.

19 En effet, nous venons de faire appel à toute une serie de circonstances qui ont poussé les études sur le langage et sur la langue vers une conception du vrai, du descriptif et du dénotatif de la langue, ce qui, depuis plusieurs années, est mis en question et critiqué par de nombreux chercheurs, philosophes, mathématiciens, linguistes et autres, qui orientent 
leurs recherches scientifiques vers des études du sens, telle que l'École argumentativiste de Paris. Ceci dit, afin d'être sensible aux critiques, il convient de signaler que tout courant de pensée en a des détracteurs et l'École argumentativiste, la conception argumentative et énonciative de la langue, en trouve aussi des critiques. Suivant les arguments de Raccah, nous essaierons d'y répondre pour appuyer la perspective sémantique que nous soutenons, i.e. la conception argumentative de la langue.

L'une des observations est liée à l'appréhension et à la description du sens. En effet, d'après Raccah le but du sémanticien est de rendre compte des contraintes que les langues mettent à la disposition des locuteurs pour effectuer des opérations sémantiques. Mais, les détracteurs de la conception argumentative de la langue se demandent alors comment y repérer lesdites contraintes qui ont un caractère subjectif. Puis, ces derniers ajoutent que le fait que les opérations impliquées dans la construction du sens soient subjectives et dépendent, d'après eux, des individus et des situations rend inacceptable de prétendre les décrire dans une étude de la langue. À ce sujet, Raccah met en relief que le fait qu'une description de ces opérations, d'après eux, ne relève pas d'une description de la langue, cela n'entraîne pas que la langue n'impose aucune contrainte sur ces opérations, principe qui d'ailleurs est plus fort que la critique. Et c'est de ces contraintes qu'il s'agit lorsque les sémanticiens parlent d'étudier ce que les langues mettent à la disposition des locuteurs pour effectuer ces opérations. C'est de ce fait qu'il resterait alors à montrer que les langues imposent effectivement des contraintes sur ces opérations.

21 Quant à la critique selon laquelle l'objet scientifique doit être réel et identifiable, il s'agit d'un principe auquel se réfère déjà Saussure ([1916] 1972) quand il distingue la matière de la linguistique, la parole, de son objet, la langue ${ }^{13}$. En fait, cette volonté scientifique d'identifier un objet réel, n'est pas toujours présente dans le domaine des Sciences dites pures, où la conceptualisation théorique s'effectue à partir d'objets théoriques créés, conçus ou imaginés. Tel est le cas par exemple de plusieurs domaines des mathématiques, de la physique, et, tout aussi, de la linguistique, etc. C'est dans ce sens où il doit être accepté que les sémanticiens, et bien d'autres chercheurs, puissent formuler des hypothèses à l'attente d'explorer les faits à observer de la façon la plus rigoureuse possible pour en rendre compte d'une façon pertinente et scientifiquement fondée, avec tous les instruments théoriques et méthodologiques actuellement à la portée de la Science. De plus, quant à la langue, de même que l'hypothèse d'une conception subjective de la langue n'est pas, d'après certains chercheurs, démontrable, ce serait le même cas pour l'hypothèse informative de la langue qui n'est pas non plus confirmable. En fait, toute hypothèse ne peut être confirmée que par la démonstration de la récurrence de sa propre conformation, c'est même un principe scientifique acceptable. En tout cas, la réponse à cette situation de la part de Raccah pour réfuter cette possible critique, c'est de montrer que les prédictions scientifiques sur la langue qu'il formule sont confirmées par ses observations, position que de nombreux chercheurs sont prêts à valider. Cette façon d'agir est d'ailleurs la base des études en langue qui, par exemple, partent de l'élaboration d'un corpus, de la formulation de plusieurs hypothèses, de la lecture de la bibliographie sur ce sujet, puis de l'analyse, la description, l'application et la vérification des hypothèses. 


\section{Le sens dans la langue} sens qui l'ont mis à l'écart scientifique pendant des siècles, tout en essayant de le dépasser et de rendre compte de certains aspects scientifiques susceptibles de caractériser le sens et de lui attribuer la place qui lui correspond, puis nous avons fait appel à la conception argumentative et énonciative pour aborder le sens et conceptualiser la configuration de la langue. C'est dans ce sens où Raccah formule certains principes qui nous semblent essentiels pour comprendre la sémantique, ainsi :

L'empiricité de la sémantique des langues impose que les propriétés sémantiques soient établies à partir de l'observation de leurs manifestations dans les discours et les énoncés. [...] La sémantique d'une langue cherche à décrire ce qui, dans la langue, contraint l'interprétation. (Raccah 2006 : 124-125)

La sémantique a pour objectif de décrire les contraintes que les phrases des langues humaines imposent aux locuteurs et interlocuteurs de ces langues dans le processus qui les conduit à construire un sens pour les énoncés de ces phrases dans chaque situation d'énonciation. [...] Ce sont ces contraintes, indépendantes donc des situations d'énonciation, qu'on appellera signification, en réservant le terme de sens au résultat de l'interprétation des énoncés, résultat qui, lui, dépend de la situation d'énonciation et qui n'a aucune raison d'être connu à l'avance, même par le locuteur : les sens ne sont donc pas reconstruits, mais construits par les interprètes (locuteurs ou interlocuteurs). (Raccah $2006: 125$ )

phrase se laissera observer indirectement à partir de l'observation plus directe des énoncés. Quant au sens, il ne peut être observé que par les effets indirects qu'il peut produire. Parmi ces effets, il en est un que Raccah utilisera à plusieurs reprises dans ce qui suit, et qu'il lui faut donc expliciter.

Un des aspects du comportement d'un interlocuteur est lié aux efforts qu'il fait pour comprendre l'énoncé. Bien entendu, ces efforts ne sont pas directement observables, et ne sont pas, en général, utiles à la description sémantique ; sauf, pour Raccah, au moins dans deux cas :

- lorsque l'interlocuteur exprime son incapacité à comprendre l'énoncé, et

- lorsqu'on l'amène à formuler des hypothèses sur la situation, hypothèses qu'il a jugé nécessaire d'admettre pour pouvoir comprendre quelque chose. (Raccah 2011 : 127)

Ces indices observables du processus de compréhension sont sûrement restreints, mais peuvent quand-même s'avérer très utiles pour étayer ou réfuter une description sémantique. En particulier, lorsqu'il est nécessaire à l'interlocuteur de faire une hypothèse complexe sur la situation pour pouvoir construire un sens, l'observateur est amené à en inférer que ce sens n'est pas fourni par les unités de langue de la phrase, mais par la situation imaginée pour comprendre l'énoncé. signification d'un mot-de-phrase doit rendre compte des différents sens des mots-dediscours auxquels il est sous-jacent. On voit ici très clairement pourquoi le premier problème méthodologique posé en est crucial pour Raccah.

L'orientation argumentative relève, d'après l'auteur, du sens, et ne peut donc pas être décrite dans la signification des mots-de-langue. Néanmoins, en retenant la leçon de Bakhtine, on n'oubliera pas que ces mots de la langue sont habités par les discours qui les

Corela, HS-19 | 2016 
ont utilisés et que ces discours, eux, pouvaient avoir des orientations argumentatives, lesquelles peuvent avoir été transmises aux mots-de-langue sous une forme latente. Cette possibilité, qui pourrait passer pour une simple curiosité ${ }^{14}$, devient intéressante si l'on tient compte de la nécessité que les mots soient eux-mêmes porteurs d'éléments susceptibles de former des contraintes argumentatives. Reste à savoir si (et comment) une orientation argumentative latente peut contribuer à la construction d'une contrainte sur l'orientation argumentative d'un énoncé.

C'est dans le cadre que l'on vient de décrire que Raccah, héritier du cadre théorique ducrotien de l'argumentation dans la langue et de la polyphonie énonciative, propose une conception de la communication langagière qu'il va appeler la métaphore de la manipulation, visant à rendre compte de la manière dont les unités de langue contraignent la compréhension de leurs énoncés et permettent, de ce fait, de recueillir et de gérer les connaissances et, par extension, où devraient être gérables tout aussi, par exemple, les savoirs-faire des experts. Cette conception de la manipulation se développe dans la théorie que Raccah (2014) formule, à savoir la Sémantique des points de vue, théorie que nous allons aborder dans les pages suivantes.

\section{Le sens argumentatif}

Cette proposition est à contextualiser dans un cadre plus large que Raccah développe depuis des années, dans le but de formuler une théorie scientifique, qui, en tout cas, mette l'accent sur plusieurs difficultés scientifiques de la sémantique. À maintes reprises, Raccah, au long de ses écrits, insiste sur le fait que la langue n'est pas observable directement, ce qui risquerait, d'après l'auteur, de mettre en question la validité scientifique de la linguistique et notamment de la sémantique, si bien que, toujours d'après l'auteur, ce sont les hypothèses et les démonstrations qui vont assurer une conceptualisation scientifique pertinente.

Ainsi, Raccah formule, suivant Ducrot, que «le sens d'un énoncé est son potentiel argumentatif » (Raccah 2006 : 11). Pour le décrire, il conçoit les hypothèses suivantes :

- H1. L'effet sémantique d'un énoncé n'est pas dû à sa matérialité ;

- H2. Les effets observables d'un énoncé ne sont pas directement causés par l'énoncé.

Des considérations précédentes découle ce que Raccah appelle le cahier de charges de la sémantique, qui part du principe selon lequel les observables sont les énoncés et les actions des interlocuteurs et dont l'objectif est de décrire les rapports entre la forme de l'énoncé et la première étape de la chaîne causale supposée relier l'énoncé à l'action. C'est cette première étape qui sera appelée sens. De plus, l'auteur fixe plusieurs hypothèses externes, ainsi :

- l'existence d'un lien causal entre certains énoncés et certaines actions ;

- la non matérialité du lien causal (attribué à la forme de l'énoncé) ;

- au moins une étape causale non observable entre l'énoncé et l'action est considérée comme causée par cet énoncé. (Raccah 2006 : 9)

C'est alors que l'on peut se demander pourquoi Raccah parle de «manipulation»? Eh bien, Raccah dit que l'art de la parole ${ }^{15}$, tel qu'il se dessine dans ces réflexions, est bien un art et non pas une technique. D'après cela,

[...] il apparaît clairement que l'auteur d'un énoncé cherche à manipuler les destinataires de manière à leur faire construire des sens qui auront, sur eux, les 
effets que lui, l'auteur, vise à produire. Le terme manipuler ne doit donc pas être pris avec ses habituelles connotations négatives [...] : il indique un faire faire, qui est caractéristique de l'énonciation. Le caractère manipulatoire de l'art de la parole est renforcé par le fait que, tel que nous l'avons souligné avec Sylvie Bruxelles [Bruxelles et alii $1992: 61$ ], un être humain « ne peut pas faire les efforts nécessaires pour ne pas comprendre un énoncé compréhensible». (Raccah 2005a : 216)

Ainsi, Raccah indique que L manipule I pour que I construise le sens que L veut que I construise.

Dans le cadre sémantique de Raccah que l'on vient de synthétiser, il existe un concept qui, depuis dix ans, se spécifie et retient l'intérêt de nombreux sémanticiens et pragmaticiens: il s'agit du concept de point de vue, clef dans la théorie de Raccah. Le concept en tant que tel n'est pas cependant récent, il existe au moins depuis un siècle dans la terminologie philosophique, mais ce n'est que récemment qu'il devient essentiel pour l'étude, la description, l'analyse, l'appréhension et la conceptualisation de la langue. Dans les pages suivantes, nous esquisserons différentes formulations de ce concept, pour, enfin, mettre en relief la proposition de Raccah.

\section{Il était une fois le point de vue}

Dans le cadre de l'application de la théorie de l'argumentation dans la langue et de la polyphonie énonciative, certains disciples de Ducrot ont insisté, vers les années $90^{16}$, sur l'importance de développer la théorie de la polyphonie énonciative et, d'ailleurs, ils ont redéfini des concepts, tels que locuteurs, énonciateurs, voix et point de vue et, même, ils ont formulé de nouveaux concepts dans le but de préciser les instruments descriptifs de la polyphonie énonciative. Il faut cependant attendre le début du XXI ${ }^{e}$ siècle pour que la plupart des chercheurs prennent conscience ${ }^{17}$ du besoin de dépasser l'horizon de l'énonciation et insistent sur un des concepts clefs, celui de point de vue.

L'intérêt porté au point de vue surgit d'une façon diffuse lors de l'éclatement de l'unicité du sujet soulevée par l'évolution même des principes scientifiques sur le langage, la langue et le discours. Tel est le cas de Bakhtine (interdiscours et interlocution), Culioli (coénonciation), Jacques (mise en communauté de l'énonciation), Goffman (rôle de l'allocutaire), Authier-Revuz (hétérogénéité énonciative), Ducrot (un locuteur mettant en scène des énonciateurs) et autres. Dans ce large cadre disciplinaire, le concept de point de vue s'insère et évolue d'abord dans un domaine cognitif et psychosocial, éthique et esthétique, puis dans un contexte sémiotique et linguistique, touchant, à son tour, le domaine dialogique et discursif ainsi que les enjeux des théories énonciatives du langage où le point de vue se situe dans son essence même. Vu la force scientifique et la capacité méthodologique et descriptive que le concept de point de vue (PDV) acquiert dans les Sciences du langage, il serait intéressant de repérer les principales perspectives qui l'intégrent, ainsi que la définition de ce qu'il comporte. Pour cela, nous allons retenir deux domaines spécialement significatifs dans leurs approches et leurs interrelations: celui de la narratologie et celui de la linguistique.

\subsection{Le point de vue dans la narratologie}

La description de la littérature, la critique littéraire, l'analyse du discours littéraire, la théorie de la littérature vont développer de nouvelles propositions théoriques et de 
nouvelles possibilités d'appréhender la langue dans la création et production littéraires. Dans cette perspective, il se produit une mise en relief spécifique du concept de point de vue. En effet, certains auteurs tels que Bres ou Rabatel, vont réfléchir aux fondements de la conception pour approfondir la connaissance des enjeux des points de vue dans le récit, dans le discours et dans la langue. D'ailleurs, dans sa critique des travaux structuralistes sur le récit, Bres (1994) signale que le récit ne se réduit pas à un ensemble monologique de structures décontextualisées renvoyant à un sens immanent, à une structure profonde, refusant toute dimension "psychologisante » et / ou « sociologisante ». Par rapport aux études de Bakhtine et de Labov, Bres insiste sur la dimension sociohistoriquement construite du sens qui provient des rapports pratiques des hommes entre eux et avec le monde. De plus, Bres (1994) fait une lecture critique de Greimas (narrativité / sens), de Ricœur (narrativité / temps) et de Labov (narrativité / intéraction verbale). Il indique l'échec de la sémiotique greimassienne dû à un isomorphisme exagéré entre les structures narratives et les structures actancielles ainsi qu'au fait de situer l'agir en dehors de l'analyse. Quant à l'herméneutique de Ricœur, Bres explique que bien que Ricœur (1983) fasse constamment appel à l'acte de raconter, en fait, il considère surtout le produit fini de la narration sans approfondir la question du temps de l'acte de raconter. En ce qui concerne Labov, inscrit dans le socio-linguistique et dans la pragmatique, la critique de Bres se situe par rapport à la définition du récit en tant que successivité des propositions strictement conforme à la succession chronologique des événements, sans entrer dans une analyse sémantique complexe de la configuration narrative. Dans la formulation bressienne, faisant appel aux recherches de Bakhtine et de Labov, l'auteur signale que «l'on atteint jamais le sens des choses, mais le sens donné aux choses " (1994 : 33), en insistant sur le principe que

le sens advient au langage du rapport de l'homme au monde; mais, parallèlement, les rapports de l'homme au monde passent par le langage. La relation entre langage et monde n'est donc pas mécaniste mais dialectique. Le langage ne décalque pas le monde : il le découpe selon le travail de l'homme. (Bres 1994 : 33)

Le sens du récit s'établit alors sur la dimension dialogique qui émerge d'autant plus dans le cadre du récit oral en situation dialogale, mais qui se constitue aussi dans le cadre de récits littéraires écrits. D’après Bres "le narré n'est pas logiquement antérieur au narrant; il est bien plutôt le produit de la fonction référentielle du langage dans son intéraction avec la praxis» (ibid. : 34) et que, dans la perspective interactionnelle des récits, leur anthropomorphisation "est la mise en spectacle par laquelle l'homme se représente les rapports praxis transformatrice / praxis linguistique » (ibid. : 36).

C'est aussi Rabatel qui, de sa part, va déveloper une narrativité de l'action, une pragmatique énonciative des textes littéraires, tel qu'il l'indique, où "les relations énonciatives à l'œuvre dans le récit ne sont pas seulement la mise en scène d'un texte pré-établi ou la manifestation de l'intentionnalité toute puissante de l'écrivain » (Rabatel 2007 : 348). Elles fonctionnent, d'après Rabatel, comme des didascalies, « des indications scéniques de nature procédurale, indiquant au destinataire comment s'approprier le texte, à partir de quels centres de perspective (tel ou tel acteur, actant ou narrateur), comment penser leurs relations, afin de faire émerger une signification co-construite par le lecteur, sur la base des instructions du texte et des choix d'empathisation effectués par le lecteur » (Rabatel 2007 : 348). Rabatel « opère ainsi un décentrement majeur : l'unité du récit n'est plus du côté du raconté, de l'énoncé, de ses structures, mais du côté de son énonciation, et de sa coénonciation pour ce qui relève de la part du lecteur » (Rabatel 2007 : 348). 
40 1946 lors de la parution du Temps et Roman de Poullion, permet d'appréhender les personnages d'une façon plus minutieuse et de déployer l'autorité du narrateur. Le PDV est lié à la prise en charge des informations narratives. En fait, c'est bien le narrateur qui, d'après Genette (Rabatel $2003: 10)$ raconte et a le choix de représenter les événements de la diégèse à partir de l'instance narratoriale (focalisation zéro), de l'instance actoriale (focalisation interne) ou à partir d'une instance sans foyer identifiable, source de la focalisation externe pour les cas où le récit se limite à un enregistrement «sans commentaires » de ces derniers. Ce cadre génettien est resté très prégnant chez les narratologues y compris chez certains linguistes se réclamant d'une narratologie énonciative. Ceci dit, plus les propositions ducrotiennes avancent dans le cadre d'une polyphonie énonciative, plus se produit, dans les études de narratologie, une fondamentation linguistique des instances narratologiques. La base de toute analyse n'est pas fixée à partir d'une relation de perception, associée principalement à des processus mentaux, mais à partir de marques linguistiques dans le discours littéraire. Dans ce cadrelà, un des chercheurs, entre autres, à spécifier la notion de point de vue, c'est Rabatel (2008a, 2008b) qui met en valeur la théorie de Ducrot au détriment de la formulation de Genette. C'est ainsi que Rabatel, sur la base d'une approche énonciative des différents points de vue, cherche à determiner le point de vue à partir d'un faisceau de marques linguistiques. Il expose les trois modalités des points de vue, à savoir le point de vue représenté ("Je suis celui qui perçoit / pense à la même place que moi »), le point de vue narrativisé ou raconté ("Je suis celui qui raconte à la même place que moi ») et le point de vue asserté ("Je suis celui qui parle / pense à la même place que moi »). Un tel choix théorique dépasse l'approche immanentiste du récit pour s'appuyer sur une analyse intéractionnelle de la narration, inscrite elle-même dans le cadre de l'analyse de discours, développée par Maingueneau (2004), à propos de l'analyse des textes littéraires, et par Amossy (2008), notamment pour l'analyse de la dimension argumentative indirecte à l'œuvre dans les récits. Rabatel réussit ainsi à dégager les différents mécanismes linguistiques à l'œuvre dans la construction des différentes modalités du PDV qui, tel que l'indique l'auteur, au-delà des différences syntaxiques et sémantiques, et indépendamment de leur gradient de mimétisme et de réflexivité, se caractérisent par leur dialogisme et leur dimension interactionnelle. Ces points de vue participent à la construction de la sphère des personnages, de celle du narrateur, et invitent le lecteur à comprendre les mécanismes linguistique et esthétique, ainsi que les enjeux dramatiques et idéologiques. L'approche multiforme de PDV demande ainsi une révision scientifique nécessaire de la narratologie, telle au moins qu'elle est formulée par Genette $(1972,1983)$ et acceptée, à l'époque, par la communauté scientifique ${ }^{18}$.

Dans l'approche du PDV de Rabatel, inspirée de Ducrot 1984, tel que l'auteur l'affirme :

le locuteur est l'instance qui profère un énoncé, selon un repérage déictique ou anaphorique, tandis que l'énonciateur, proche du sujet modal de Bally, assume l'énoncé dans la mesure où les évaluations, qualifications, modalisations et jugements sur les objets du discours sont donnés à travers sa subjectivité et sont validés / validables par ce dernier. Souvent, les énoncés reposent sur un syncrétisme locuteur / énonciateur, comme à chaque fois que le locuteur pense ce qu'il dit et dit ce qu'il pense - ou feint de dire ce qu'il pense. Mais le locuteur / énonciateur primaire peut aussi développer dans son discours des PDV qu'il ne partage pas nécessairement, comme dans l'ironie, les hypothèses, le discours indirect libre, ou encore dans les énoncés délocutés qui expriment un PDV, dans les narrations hétérodiégétiques au passé. (Rabatel 2007 : 349) 


\subsection{Le point de vue dans la linguistique} linguistiques instaurées, vehiculées et soutenues dans et par des figures discursives possibles, telles que sujet, locuteur, énonciateur, voix, angle de vue ou point de vue, entre autres. Nous considérons alors que le PDV langagier est inscrit dans la langue et se déploie dans le discours moyennant des dynamiques linguistiques par lesquelles une figure ${ }^{19}$ discursive, plus ou moins singulière ou collective, privée ou publique, réelle ou songée, inscrit, appréhende ou envisage ${ }^{20}$ une situation ou un objet discursif, repérable ou langagier.

La figure discursive, responsable sémantiquement de l'appréhension, construction ou référenciation de la situation ou de l'objet discursif, exprime un / son / des points de vues d'une façon plus directe (commentaires explicites), ou plus indirecte, par la configuration sémantique implicite (orientation, idéologies, convocation, variables, choix, combinaison, actualisation... de principes et d'instruments linguistiques, etc.). Ces phénomènes agissent dans tous les cas, depuis les principes langagiers, linguistiques et discursifs, intrinsèquement subjectifs, aux choix apparemment objectivants, depuis les traits les plus implicites jusqu'aux marques les plus explicites. L'idée de point de vue n'existe pas sans une certaine problématique, dès le moment où il existe différentes facettes, processus et actions discursives qui lui sont liées et sont susceptibles de nuancer un type de conception de sens : pensées, attitudes, actes, illocutoires, présupposés, posés, positions, paroles. De là une considération du sens dont la base peut répondre plutôt à une conception qui est appelée "attitudinale ${ }^{21}$ ou à une conception dite polyphonique «musicale » et même à ce que plus récemment Carel et Ducrot (2009) posent comme une conception triadique de la polyphonie, qui s'établit sur des triplets.

\subsection{1. À l'origine des points de vue}

Dans le cadre de la linguistique, nous rappelons que c'est Ducrot, en effet, à partir des années 1970, en héritage probablement d'un concept présent, autrement, dans la théorie bakhtinienne, qui va reprendre le concept de point de vue, cette fois-ci appliqué au sens de l'énoncé, quand il expose la Théorie polyphonique de l'énonciation (1984). Il est à signaler cependant que Ducrot ne développe pas suffisamment à cette époque ${ }^{22}$ le concept de point de vue en tant que tel et utilise, fondamentalement, deux concepts à cette époque et jusqu'aux années 2000, celui de locuteur et celui d'énonciateur. En fait, il utilise le concept de point de vue dans la description de la polyphonie, mais ce concept ne constitue pas une figure discursive, au même point que le locuteur et l'énonciateur. Ducrot distingue le locuteur des énonciateurs et, en effet, c'est le concept d'énonciateur qui, à son tour, est lié en quelque sorte, pour ne pas dire identifié à certains moments, au PDV, quand il dit :

J'appelle «énonciateurs" ces êtres qui sont censés s'exprimer à travers l'énonciation, sans que pour autant on leur attribue des mots précis; s'ils " parlent », c'est seulement en ce sens que l'énonciation est vue comme exprimant leur point de vue, leur position, leur attitude, mais non pas, au sens matériel du terme, leurs paroles. (Ducrot 1984 : 204)

Puis, Ducrot ajoute : 
Le locuteur, responsable de l'énoncé, donne existence, au moyen de celui-ci, à des énonciateurs dont il organise les points de vue et les attitudes. Et sa position propre peut se manifester soit parce qu'il s'assimile à tel ou tel des énonciateurs, en le prenant pour représentant (l'énonciateur est alors actualisé), soit simplement parce qu'il a choisi de les faire apparaître et que leur apparition reste significative, même s'il ne s'assimile pas à eux. (Ducrot 1984 : 205) 
autres. Des travaux de recherche sur l'énonciation et sur l'acquisition énonciative du Je, dès la naissance, mettent l'accent sur l'inscription du locuteur dans le monde au travers la langue. De plus, il est à signaler, tel que l'indique Rabatel (2003), que le paradigme énonciatif et d'analyse du point de vue croise trois grands cadres théoriques :

- l'empathie (Kuno, Forest, Cantrall, Kuroda, Zribi-Hertz, etc.) ;

- les sources évidentielles (Tasmowski, Dendale, Vogeleer, etc.) ;

- le discours rapporté (Danon-Boileau, Banfield, Mattia, Rabatel, Rosier).

D'autre part, il convient de signaler que certains linguistes ont fait des points de vue un instrument clef de leur conception et description de la langue. Quant à Nølke, il a insisté clairement sur les points de vue. En effet, le parcours du concept de point de vue est repris et spécifié par Nølke, dans la théorie Scalopine. L'auteur fait du point de vue un concept central et identifiable. En effet, l'auteur considère que la configuration énonciative fait partie du sens, elle est un élément de la description sémantique de l'énoncé, dira-t-il (2001a, 2001b). L'énoncé est conçu comme le résultat de l'énonciation, comme une image de cette énonciation. Il s'ensuit que la configuration sémantique renferme des images des instances énonciatives et notamment du locuteur qui, en effet, est l'élément constitutif de la configuration. La configuration est construite, selon Nølke, par le locuteur et se compose de quatre éléments, à savoir, le locuteur lui-même et trois types d'entités construites par celui-ci. Le locuteur, responsable de l'énonciation et qui construit les éléments dont se compose la configuration polyphonique; les points de vue, entités sémantiques porteuses d'une source qui sont des êtres abstraits appelés les énonciateurs (les énonciateurs étant des variables, qui cherchent à être saturées par les interprètes); les êtres discursifs, entités sémantiques susceptibles de saturer les énonciateurs; liens énonciatifs, qui relient les êtres discursifs aux points de vue. La configuration construite par le locuteur se compose donc de PDV reliés aux êtres discursifs par différents liens. Tous ces éléments doivent être marqués dans la signification, mais ils ne le sont pas forcément. Les éléments marqués forment la structure-polyphonique, tel que l'indique l'auteur.

L'auteur introduit une structure interne, quand il dit : « chaque énoncé contient au moins un point de vue simple dont le contenu sémantique est posé " (Nølke, Fløttum et Norén 2004 : 249). Il va même plus loin, il complexifie le concept en proposant le point de vue simple, le point de vue hiérarchique et le point de vue relationnel, tout en entraînant, semble-t-il, la disparition de la notion d'énonciateur. C'est ainsi que Nølke formule les points de vue de la façon suivante :

Les pdv sont (normalement) marqués dans la signification; ils forment le « corps » de la structure-polyphonique. On pourra distinguer trois types de pdv selon leur structure interne : les $p d v$ simples sont indépendants des autres pdv, ils constituent les éléments atomiques de la construction polyphonique et tout énoncé contient au moins un pdv simple, qui se compose d'un contenu sémantique et d'un jugement porté sur ce contenu, qui peut être propositionnel, argumentatif ou autre; puis deux types de $p d v$ complexes; les $p d v$ hiérarchiques (permettent de faire porter des jugements, extérieurs, sur d'autres jugements) se composent de points de vue simples ou complexes organisés selon une structuration hiérarchique et les $p d v$ relationnels, ils relient des pdv simples ou complexes entre eux. On aura notamment des pdv relationnels dans les phrases renfermant des connecteurs; un quatrième type de points de vue s'ajoute au moment de l'énonciation, les pdv stratificationnels qui sont attachés à la focalisation neutre et qui, en tant que tels, sont eux aussi marqués au niveau de la phrase. (Nølke, Fløttum et Norén 2004 : 46) 
54

formulent, en 2002, une hypothèse selon laquelle les emplois de donc reflèteraient des jugements élémentaires. Ainsi la TBS considère que les mots préfigurent la signification, indépendamment de la manière dont un référent a été désigné: toutefois, ce fonctionnement des particules consécutives ne remet pas en cause la dimension polyphonique des "articulateurs" qui, à l'instar de mais, marquent la polyphonie en renvoyant possiblement à un énonciateur distinct du locuteur. La TBS conçoit les contenus comme des "argumentations", c'est-à-dire comme des enchaînements d'énoncés liés par un connecteur. La liaison entre l'angle de vue et le point de vue pourrait être représentée par des enchaînements en donc, qui, selon la conception de l'enchaînement argumentatif, marquent, non pas l'association de deux notions indépendantes, mais l'interdépendance sémantique de deux expressions, dont chacune signifie à travers l'autre.

C'est ainsi que Carel et Ducrot (2009) proposent, à partir de la conception polyphonique de l'énonciation de 1984 élaborée par Ducrot dans Le dire et le dit, une nouvelle conception polyphonique, dite conception triadique de la polyphonie, du moment où elle s'établit sur la base d'un triplet. Les auteurs l'expliquent de la façon suivante :

Dans chacun de ces triplets, on trouve les trois éléments suivants : d'une part, une attitude du locuteur de l'énoncé (présente déjà dans la conception «attitudinale » de la polyphonie et absente de la conception « musicale » puisque celle-ci refuse de représenter le locuteur de l'énoncé dans la signification). Ensuite un contenu (présent à la fois dans la conception "attitudinale» et dans la conception « musicale »), et enfin un " énonciateur » (absent de la conception « attitudinale » et qui, à certaines et importantes différences près, [...] correspond à la voix productrice du discours dans la conception « musicale»). (Carel et Ducrot $2009: 10$ )

Il s'agit de la placer dans la signification, à côté des attitudes du locuteur et des contenus. Cette troisième instance qui, pas plus que les deux autres, n'est de type référentiel et ne peut être vue comme allusion à des individus. Elle marque seulement une certaine façon de garantir le dit, un certain ton pour le présenter, et l'exigence corrélative d'un ton particulier pour le réfuter. Dans ce cas, il n'y a pas de limite inférieure au PDV, ce dernier, embryonnaire, existe en langue, en fonction de l'orientation argumentative des lexèmes (Carel et Ducrot 1999). Dans le cadre de cette version triadique de la polyphonie énonciative, qui est présentée comme nouvelle, il est à signaler que différents auteurs ont déjà noté, depuis des années (Tordesillas 1998), les circonstances de la polyphonie exposées par les auteurs, ainsi que le développement discursif lié au locuteur, tel que «la prise en charge » (poser), attitude, « accord » (présupposés), énonciateur, «l'exclusion » (énoncé négatif), contenu. Il faut dire que des concepts d'«angle», "point de vue », «ton», «Personne» et «Monde» viennent enrichir la proposition théorique polyphonique.

\section{Le concept de point de vue comme fondement de la langue : polyphonie et argumentation}

57 Le principe selon lequel le point de vue est un fondement langagier, tel que nous le formulons depuis des années, est soutenu par Tordesillas (2004b) dans le cadre de la Sémantique argumentative et énonciative qu'elle propose depuis les années 90 . Tordesillas $(1992,1998)$ en formulait déjà trois figures discursives inscrites dans l'énonciation dans la 
langue : locuteur, énonciateur, point de vue, ayant un regard, une visée et un but, puis, elle formulait des relations entre les différentes figures, ainsi prendre en charge, accorder, s'identifier, nier, rejeter. Elle distinguait déjà, dans la dynamique jouée entre les points de vues (positifs, négatifs ou neutres) liés aux topiques, des contraintes à travers, réciproquement, les énonciateurs, relations de prise / reprise des orientations (favorable, non favorable, défavorables) concernant trois processus essentiels, ainsi déployer le topos, dériver le topos, enchaîner le topos. Puis, elle formulait une nouvelle dynamique, dialogique interne, conversationnelle même, constituée par la relation entretenue entre le locuteur et les énonciateurs. En fait, d'une part, le locuteur est susceptible de présenter un ou plusieurs énonciateurs, puis, d'autre part, le locuteur est susceptible de se prononcer sur un ou plusieurs énonciateurs et, cela, en fonction de l'implication du locuteur dans le dire et le dit, de la responsabilité qu'il assume, du degré de cohérence, de cohésion et de transparence visé et lié tout aussi à la signification même intrinsèque instruite par la langue. À cela, Tordesillas (1998) ajoute qu'elle conçoit les liens en termes hirérarchiques et ayant une démarche de plus à aborder, celle qui concerne la conclusion externe. Dans ce cadre-là, le point de vue est présenté comme la signification même constitutive et contraignante de la langue, que Tordesillas définit et inscrit même dans le concept de signe :

Nous définirons alors le signe, langagier, comme tissu langagier intersubjectif comportant des points de vue discursifs dialogiques (argumentatifs et énonciatifs), ayant une tenue verbale - acoustique (orale, sonore) et / ou un graphique (écrit, visuelle) -, ainsi que des lieux discursifs communs, visant un but discursif et susceptible de construire un monde discursif, lieu de présence et d'intéraction du Je. (Tordesillas 2008 : 1948)

Le tissu langagier est susceptible d'avoir différents supports, la langue, la gestualité, l'espace, etc.

Nous avons parcouru une réflexion sur la langue pour nour situer scientifiquement dans une approche argumentativo-énonciative. Nous avons abordé certaines théories liées à la polyphonie énonciative pour en présenter le concept de point de vue et leur implication.Dans les pages qui suivent, nous allons nous situer au sein même du concept de point de vue et, avec cela, nous allons présenter une théorie linguistique où le point de vue est le pilier de sa formulation, tel est le cas de la Sémantique des Points de vue, proposée par Raccah.

En effet, Raccah va s'occuper spécifiquement du concept de point de vue, qui donne lieu à la théorie qu'il formule. Il s'agit d'un cadre théorique qui vise à construire des modèles susceptibles de décrire l'ensemble des phénomènes sémantiques, tout en rendant compte de ce caractère manipulatoire de la langue que Raccah conçoit et, dont on a parlé au long des pages précédentes. La Sémantique des points de vue s'appuie sur certaines hypothèses formulées auparavant par différents philosophes, puis sur d'autres, originales, établies par Raccah. En voici les hypothèses principales :

- l'hypothèse polyphonique, proposée par Bakhtine, puis reprise, reformulée et inscrite dans la sémantique par Ducrot;

- l'hypothèse argumentationnelle, proposée par Ducrot et adaptée, avec la formulation de nouveaux concepts, à la description du lexique par Raccah ;

- le modèle des topoi, formes topiques et champs topiques lexicaux, proposé à un certain moment dans le cadre de l'Argumentation dans la Langue et, que Raccah spécifie, développe et rend plus technique. 
61

Raccah pose que la description sémantique d'une langue humaine est la description de l'ensemble des contraintes que les mots et les structures de cette langue imposent à la construction des sens des énoncés de cette langue. De manière plus spécifique, il indique que ces contraintes sur la construction du sens comportent que les discours visent à faire adopter des points de vue et, pour ce faire, s'appuient sur d'autres points de vue. Le cadre théorique que l'auteur présente combine deux concepts de points de vue, l'un provenant de la polyphonie bakhtinienne, l'autre provenant de l'argumentation ducrotienne et, ceci, dans un modèle qui rend compte des manipulations idéologiques que, selon Raccah, les langues permettent aux discours d'effectuer. Voyons les principales bases scientifiques sur lesquelles se fonde le cadre théorique proposé par Raccah.

Nous avons déjà signalé la double origine de la conception polyphonique qui inspire Raccah : Bakhtine et Ducrot. La conception polyphonique du discours a été présentée et systématisée par Bakhtine (Voloshinov ([1929 / 1963] 1977), dès la fin des années vingt, et utilisée par l'auteur pour des analyses littéraires approfondies (Bakthine [1923 / 1963] 1970). Cette conception du discours et du texte littéraire est, actuellement, couramment acceptée et, l'apport de Bakhtine et de son école aux études littéraires est largement reconnu et appliqué.

La polyphonie discursive de Bakhtine, liée au concept épistémologique de dialogisme, concerne ce que Raccah appelle les « mots-de-discours ». Ainsi Raccah explique :

l'idée générale est que, lorsque nous parlons une langue que nous maîtrisons bien (par exemple notre langue maternelle), les mots que nous employons gardent, pour nous, les traces des discours dans lesquels ils ont été employés, et à travers lesquels nous avons acquis l'usage de ces mots (à travers lesquels nous avons, en quelque sorte, fait connaissance de ces mots). Ce phénomène apparaît aussi dans la compréhension des discours : lorsque j'entends le mot-de-discours $M$ (occurrence particulière du mot-de-langue $M$ ) dans un discours $D$, ma compréhension de $D$ ne peut pas ne pas subir l'influence de la manière dont j'ai compris les mots-dediscours $M_{1}, M_{2}$, etc. (occurrences antérieures du même mot-de-langue $M$ ) dans les discours $D_{1}, D_{2}$, etc. qui ont contribué à ma familiarisation avec ce mot. Cette particularité de la compréhension permet d'ailleurs d'expliquer le rôle important de la littérature dans l'évolution des langues. (Raccah 2008 : 77)

Bakhtine lui-même insistait sur l'idée que ce qu'il proposait ne concernait que les discours sans être destiné à s'appliquer aux langues. C'est justement cette application dont va s'occuper la conception polyphonique de la langue établie, il y a plus trente ans, par Ducrot.

Ducrot, dans le cadre de la Théorie de la polyphonie énonciative, reprend les concepts de dialogisme et de polyphonie bakhtiniens et a le grand mérite de les inscrire dans la langue elle-même. Nous l'avons déjà brièvement présentée précédemment, donc, dans ce qui suit, nous l'aborderons juste dans la mesure où Raccah adapte le modèle polyphonique à la sémantique des points de vue.

Le locuteur, responsable d'un énoncé, attribue des points de vue à des énonciateurs. Pour chaque point de vue, chaque énonciateur, il indique sa propre attitude : accord, opposition ou identification ${ }^{24}$. Une description polyphonique d'une phrase indique ainsi, selon Raccah (2006), les contraintes que cette phrase impose sur les points de vue évoqués par ses énoncés, sur l'attribution de ces points de vue, et sur l'attitude du locuteur vis-à-vis des énonciateurs porteurs de ces points de vue. Au niveau de l'analyse de la phrase, seules les contraintes peuvent être décrites : c'est au niveau de l'analyse de l'énoncé ou du discours que les points de vue eux-mêmes sont explicités. Les mots [-de-langue] et les syntagmes 
contraignent les points de vue attribués aux énonciateurs, les structures de cette attribution, ainsi que les choix concernant l'attitude du locuteur vis-à-vis des points de vue qu'il attribue aux différents énonciateurs. La manière de décrire les contraintes sur les points de vue va de paire avec l'application du concept de champ topique à la description lexicale ; la polyphonie dans la langue, quant à elle, décrit les contraintes sur les attributions de points de vue et sur l'attitude du locuteur vis-à-vis des énonciateurs qu'il évoque.

Quant à l'argumentation, se fondant sur le fait qu'une argumentation vise à faire adopter un point de vue tout en supposant admis d'autres points de vue, la Sémantique des Points de Vue propose de décrire, dans un système unifié, et les orientations argumentatives et les points de vue, assignant pour tâche à la sémantique de décrire les contraintes que les unités linguistiques imposent aux points de vue visés par les énoncés, mais aussi aux points de vue supposés par ces énoncés. Il faut en effet distinguer les points de vue nécessaires à la compréhension des points de vue obtenus par la compréhension: les premiers fonctionnent comme des 'présupposés argumentatifs', des sortes de conditions d'interprétabilité, alors que les seconds résultent de l'interprétation.

\section{Caractéristiques des points de vue}

Pour aborder en profondeur le concept de point de vue et connaître d'une façon plus précise son essence même énonciative et argumentative dans la langue, sa signification, son rôle, sa fonction, sa nature, Raccah met en valeur les particularités dudit concept, dans les différentes facettes qu'on peut lui attribuer. C'est ainsi qu'il expose une analyse qui permet de cerner certaines caractéristiques sur les contraintes que les points de vue comportent. Nous allons les aborder à la suite.

\subsection{Contraintes sur la nature des points de vue}

Raccah explique que certaines des contraintes que les langues imposent aux points de vue visés par les énoncés proviennent des articulateurs: il s'agit principalement de contraintes sur la forme des argumentations et le but consiste à faire ressortir les instructions que ces articulateurs ${ }^{25}$ donnent à l'interprète et à choisir, parmi ces instructions, celles qui sont indépendantes des situations d'énonciation et des points de vue des interprètes. Par ailleurs, Raccah indique qu'il existe d'autres mots qui imposent des contraintes sur les points de vue et, notamment, sur la nature des points de vue. Cette hypothèse doit être circonscrite de manière très précise, car il est clair que, à son avis, les mots-de-langue déterminent les points de vue de la phrase, sans pour autant déterminer les orientations argumentatives des énoncés des phrases que l'on peut construire avec eux et qui peuvent dépendre aussi des situations d'énonciation et des situations d'interprétation. Il observe que ces points de vue au moyen desquels il faut décrire ces mots-de-langue ne sont pas systématiquement les mêmes que les points de vue de tous les énoncés qui les contiennent. Ainsi, même si un mot-de-langue doit être décrit en faisant référence par exemple à un point de vue positif, il n'en va pas de même nécessairement pour l'énoncé. Dans ce sens, Raccah insiste sur le fait qu'il ne faut pas assimiler la notion de points de vue évoqués par les mots-de-langue avec celle de points de vue évoqués par les énoncés, ce qui n'empêche de prendre en compte le fait que des points de vue exprimés directement par des mots-de-langue contraignent les points de vue exprimés par les 
énoncés qui les utilisent, sans pour autant s'assimiler à eux. Cette situation nous renvoie à la nature des points de vue. D'autre part, Raccah met en relief que le fait de voir une entité selon un certain point de vue contraint le point de vue que l'on peut avoir sur d'autres entités. Cette propriété peut être rendue par une structuration récursive des points de vue. Raccah représentera cette structuration par des chaînes de couples ordonnés, dans lesquels le premier terme désigne l'entité dont on parle et le second terme désigne le point de vue que l'on a sur cette entité. C'est dans ce sens où Raccah (2011) dit : si " je " vois $\mathrm{Y}$ avec le point de vue $\mathrm{Z}$ et si " ma " manière de voir $\mathrm{X}$ dépend de " ma " manière de voir $\mathrm{Y}$, alors, " ma " manière de voir $\mathrm{Z}$ influencera " ma " manière de voir $\mathrm{X}$. Raccah formula alors l'écriture suivante : $<\mathrm{X},<\mathrm{Y}, \mathrm{Z}>>$.

\subsection{Contraintes evaluatives élémentaires des points de vue} certaines entités sont vues de manière positive (ou négative) sans qu'il soit nécessaire ni même possible de recourir à l'intermédiaire d'autres points de vue pour construire ou justifier la manière de voir ces entités. Il s'agit de points de vue subjectifs élémentaires, qui peuvent s'exprimer selon une convention, en introduisant deux primitives : une pour les points de vue positifs, et l'autre pour les points de vue négatifs (par exemple, bien et mal, ou bon et mauvais, ou encore, + et -). L'auteur formule ces évaluations élémentaires par des couples ordonnés dont le premier terme est l'entité et dont le second terme est l'une de ces deux primitives, du type: un point de vue positif élémentaire sur $\mathrm{X}$ s'exprimera $<\mathrm{X}$,bien $>$ (ou $<\mathrm{X}$,bon $>$ ou encore $<\mathrm{X},+>$, selon les symboles choisis pour les primitives). Cette propriété permet de garantir que les chaînes de points de vues ne seront pas infinies, et peuvent arriver jusqu'à trois enchâssements : $\langle\mathrm{X},<Y,<\mathrm{Z}$,bien»>> .

Cette caractéristique propre aux points de vue élémentaires marque les mots de la langue. D'ailleurs, quand le mot est marqué par le positif, Raccah va le désigner comme euphorique, alors que quand il est présidé par le point de vue négatif, il sera dit dysphorique . Certains mots, les euphoriques et les dysphoriques, imposent des contraintes évaluatives élémentaires, d'autres mots contraignent la manière de voir une entité en s'appuyant sur la manière de voir une autre entité. Ainsi, le mot français "honnête", qualifié d' euphorique, indique que le locuteur de tout énoncé d'une phrase le contenant se présente comme portant un jugement positif sur la personne. De son côté, le mot "malhonnête ", dysphorique, entraîne l'attribution au locuteur d'une énonciation comportant un jugement négatif. Raccah fait appel à une autre catégorie de mots, celle dont le jugement positif ou négatif associé aux mots dépend d'une position idéologique, explicite ou implicite. Tel est le cas, par exemple, du mot « conservateur » qui évoque un jugement négatif quand il est employé dans un cadre idéologique dit de gauche: dans un cadre opposé, ce mot n'entraîne pas un jugement négatif.

Il est à signaler qu'un calcul sématique des points de vue serait envisageable du moment où l'hypothèse sur les jugements positifs et négatifs à laquelle on vient de faire appel est posée comme scientifiquement valable. Il devrait alors être possible de programmer ce type de signification. C'est ainsi que Raccah parle de « mini-programmes » argumentatifs, faisant à la fois appel au concept de champ topique lexical. En effet, Raccah indique :

Une première utilisation des propriétés que nous venons de voir consiste à élaborer des moyens pour 'calculer' la force idéologique des mots-de-discours : en combinant les contraintes imposées par les articulateurs avec celles imposées par d'autres mots, comme, par exemple, les euphoriques / dysphoriques on peut déterminer, par 
un calcul précis, les points de vue implicites qu'il est nécessaire d'attribuer autres mots-de-discours utilisés dans l'énoncé observé, pour que ce dernier soit compréhensible. (Raccah 2005c : 6)

\title{
7.3. Fonctionnement général des points de vue
}

73 À ce point de l'analyse, il est intéressant de synthétiser, dans le cadre général de l'argumentation et de l'énonciation, les liens entre les différents types de contraintes, l'orientation et les points de vue. C'est dans ce sens où Raccah formule :

\begin{abstract}
Une argumentation vise à faire adopter un point de vue tout en supposant admis d'autres points de vue : la SPV propose de décrire dans un système unifié et les orientations argumentatives et les points de vue, ce qui permet de formuler, de manière homogène, les contraintes que les unités linguistiques imposent aux points de vue visés par les énoncés, mais aussi aux points de vue supposés par ces énoncés. Il faut en effet distinguer les points de vue nécessaires à la compréhension des points de vue que cette compréhension permet d'obtenir : les premiers constituent ce que nous avons appelé les 'présupposés argumentatifs', alors que les seconds résultent de l'interprétation. (Raccah $2011: 168$ )
\end{abstract}

74 À cet effet, nous reprenons certains aspects que l'on vient de présenter dans les paragraphes ci-dessus pour rendre compte de la distinction qu'établit Raccah entre :

- les contraintes sur les relations entre points de vue, portées principalement par les articulateurs (opérateurs ou connecteurs) ; et

- les contraintes sur la nature des points de vue, portées principalement par les mots 'ordinaires' du lexique.

Puis, dans cette dernière catégorie, il distingue à nouveau entre :

- les contraintes évaluatives élémentaires, portées par certains mots (les 'euphoriques' et les 'dysphoriques') ; et

- les contraintes évaluatives constituées d'une chaîne de points de vue, contraintes qu'il appelle 'mini-programmes' et qui sont portées par la plupart des mots et des syntagmes de chaque langue.

76 À cela, Raccah spécifie que, pour chacune de ces contraintes, il faut prendre en compte son statut posé ou présupposé : tel point de vue, telle relation entre points de vue, telle évaluation, est-elle présentée par le locuteur tel que son discours défend, ou est-elle présentée comme quelque chose que les destinataires sont censés admettre avant même le discours du locuteur?

77 Par ailleurs, il est intéressant de faire appel à un aspect ducrotien dont parle l'auteur, il s'agit de la notion de force idéologique, tout en distinguant la force idéologique des motsde-discours utilisés dans les énoncés de la force idéologique des mots-de-langue correspondants. Pour son repérage et son calcul, les instruments provenant de la description des articulateurs en deviennent pertinents, car ils permettent de décrire les autres mots-de-langue.

\section{Esquisse du modèle topique dans la Sémantique des points de vue}

78 Nous pouvons remarquer que le principe de contrainte est essentiel dans le cadre théorique de la SPV. D'ailleurs, nous observons que Raccah cherche à établir les différents types de contraintes et à les formuler de manière unifiée dans le modèle topique de la Sémantique des Points de Vue qu'il propose, modèle que nous allons synthétiser à la suite. 


$$
\text { représentés par des champs topiques élémentaires, ainsi : }
$$

- Si X représente une entité, alors $<\mathrm{X}$, bien $>$ et $<\mathrm{X}$, mal $>$ sont deux champs topiques élémentaires.

Les points de vue non élémentaires, à leur tour, sont représentés par des champs topiques complexes, définis récursivement comme suit :

- Si X représente une entité et si CT est un champ topique quelconque, alors $\langle\mathrm{X}, \mathrm{CT}>$ est un champ topique complexe.

Enfin, d'après ce que l'auteur définit, nous pouvons constater que :

- un champ topique est soit $\odot$ un champ topique élémentaire, soit un champ topique complexe.

Les points de vue que les mots-de-langue suggèrent s'expriment par des champs topiques lexicaux, qui ont donc la particularité de permettre de décrire un point de vue sur une entité au moyen de points de vue sur d'autres entités. Les énoncés, à leur tour, mettent en relation des champs topiques. Il convient de donner quelques caractéristiques de ces relations.

Ainsi, une relation graduelle entre deux champs topiques constitue une règle d'inférence graduelle, appelée topos ${ }^{26}$. Raccah montre qu'un topos est une catégorie de garants d'argumentation, indiquant la prise en compte d'une corrélation entre les manières de voir deux entités. La rhétorique a montré que ces garants sont présentés par les locuteurs comme généraux et partagés par l'ensemble de la communauté linguistique (intersubjectivité). Ces caractéristiques se répercutent sur les topoi, qui sont donc, eux aussi, présentés comme généraux et comme partagés. Étant une catégorie de garants, le topos a, en outre, une structure graduelle et sa forme générale peut encore être décrite par: //plus (ou moins) A est $P$, plus (ou moins) $B$ est $Q / /$ où $P$ et $Q$ sont les champs topiques $<\mathrm{X}_{1}, \mathrm{Y}_{1}>$, et $\left\langle\mathrm{X}_{2}, \mathrm{Y}_{2}>\right.$, respectivement.

Il est intéressant de remarquer que la donnée d'un champ topique complexe permet de déterminer un topos unique, par l'application d'un processus simple pouvant systématiquement être appliqué : on appellera ce topos 'le topos canoniquement associé au champ topique'. $\mathrm{Si}\langle\mathrm{X}, \mathrm{Y}\rangle$ est un champ topique, alors $/ /<\mathrm{X}, \mathrm{Y}>\mathrm{Y} / /$ est le topos canoniquement associé à $\langle X, Y>$. Raccah dit alors qu'un mot-de-langue que l'on décrit en utilisant le champ topique $<\mathrm{X}, \mathrm{Y}>$ cristallise le topos $/ /<\mathrm{X}, \mathrm{Y}>, \mathrm{Y} / /$.

Dans un topos de la forme //plus (ou moins) A est $P$, plus (ou moins) B est $Q / /$, la prise en compte de la corrélation entre les deux manières de voir les entités $A$ et $B$ est discursive : rien n'empêche à un locuteur d'admettre un topos dans un discours et d'admettre le topos contraire dans un autre. Néanmoins, l'hypothèse des champs topiques lexicaux suppose que la langue 'choisit' certains topoi pour les cristalliser dans ses mots, sous forme de champs topiques. Les topoi discursifs sont alors contraints par ces champs topiques lexicaux, sans pour autant être totalement déterminés par eux.

Grâce à cette propriété du concept de points de vue qui résulte des discussions précédentes, et qui fait qu'un point de vue sur une entité peut être partiellement déterminé par un point de vue sur une autre entité, il est possible de construire récursivement, à partir de points de vue élémentaires, des points de vue plus complexes, enchâssant ces points de vues élémentaires. Le modèle topique dans la SPV de Raccah rend compte de cette propriété d'enchâssement des points de vue au moyen de champs topiques définis récursivement. 


\section{Conclusion}

88

la langue, en considérant les axiomes qui sont devenus des entraves pour le développement de nouveaux aperçus qui se sont développés au long de l'histoire de la pensée scientifique sur les dits concepts. Nous avons précisé différentes possibilités scientifiques innovatrices capables d'aborder un défi contemporain, à savoir le sens, en insistant sur la contribution de la théorie de l'argumentation et de l'énonciation. Nous avons fixé notre attention sur des principes clés pour la découverte, analyse et description du sens, tels que les caractères dialogiques et dynamiques du sens, et même de la signification, à partir des voix du discours, de la polyphonie énonciative. Enfin, nous nous sommes arrêtés sur un concept précis qui nous permet d'approfondir l'analyse de la configuration du sens du discours et de concevoir la signification de la langue : il s'agit du concept de point de vue.

Les différentes questions auxquelles nous venons de faire appel dans le cadre de la sémantique générale, sont étudiées, attentivement, par Pierre-Yves Raccah, tout en offrant à la linguistique générale contemporaine la formulation d'une théorie scientifique clef susceptible de rendre compte, d'une façon technique, avec un métalangage précis, du sens. Il s'agit de la théorie appelée par l'auteur: Sémantique des points de vue. D'après Raccah, les mots et les structures d'une langue contraignent les points de vue que le locuteur qui les emploie prétend avoir sur les entités que ces mots évoquent, ou qu'il attribue à l'un de ses énonciateurs. Il montre aussi qu'il est nécessaire d'admettre que les mots ordinaires imposent des contraintes sur les points de vue et, plus précisément, sur la nature des points de vue que les énoncés peuvent évoquer. L'auteur observe, ensuite, que les points de vue évoqués par les mots-de-discours sont contraints par les jugements que l'interprète porte sur d'autres entités que celles que les mots évoquent, et que cette caractéristique devrait apparaître dans la description des mots-de-langue. Ces derniers déclenchent, en effet, ce que Raccah appelle des 'mini-programmes' argumentatifs évoquant des points de vue qui s'appuient sur d'autres points de vue. Ces miniprogrammes, cristallisés dans le lexique, proviennent pour l'auteur de biais sociocognitifs, qui dépendent de la culture, du mode de vie, des aspirations de chaque communauté linguistique. Sans pouvoir entrer dans les details ${ }^{27}$, nous dirons que la Sémantique des points de vue propose un formalisme unifié, les champs topiques dans lequel les points de vue lexicaux, les contraintes des articulateurs et les points de vue discursifs peuvent être décrits. Enfin, on peut ajouter une richesse de la théorie raccahienne dès le moment où elle comporte un principe intéressant qu'il développe dans plusieurs recherches. C'est que si l'on admet l'hypothèse d'une identité de structures entre la gestion des connaissances et la construction du sens (quel que soit l'origine causale que l'on veut attribuer à cet homomorphisme), les points de vue que les discours (et les textes) des experts révèlent correspondent à un modèle efficace de leur expertise, moyennant un tri fondé sur la connaissance des propriétés sémantiques de la langue que l'expert utilise. La théorie des points de vue offre ainsi plusieurs champs de recherche essentiels pour la connaissance des sciences du langage, car, d'une part, elle s'occupe des fondements scientifiques de la sémantique et, d'autre part, elle constitue une application de la linguistique pour la gestion sémantique des connaissances générales et expertes. Ainsi, la Sémantique des points de vue est un modèle sémantique de qualité qui peut servir à une 
conception, description et analyse sémantiques profondes de toute interrelation et interaction langagières. Nous tenons donc à remercier à Pierre-Yves Raccah pour son implication à la science en tant que chercheur et pour sa recherche poussée, bien fondée et innovatrice qui enrichit les fondements scientifiques et contribue au développement de la sémantique et de la linguistique générales et des sciences du langage.

\section{BIBLIOGRAPHIE}

Anscombre Jean-Claude, « Délocutivité benvenistienne, délocutivité généralisée et permormativité », Langue française, vol. 42, n 1, 1979, pp. 69-84.

Anscombre Jean-Claude et Ducrot Oswald, «L'argumentation dans la langue », Langages, vol. 10, $\mathrm{n}$ o 42, 1976, pp. 5-27.

Anscombre Jean-Claude et Ducrot Oswald, L'argumentation dans la langue, Liège, Mardaga, 1983. Amossy Ruth, « Argumentation et Analyse du discours : perspectives théoriques et découpages disciplinaires ", Argumentation et Analyse du Discours nº 1, 2008, pp. 2-15.

Bakhtine Mikhaïl, Problemy tvorchestva Dostoevskogo, Leningrad, 1929, 2 ème ed. rev. Probemy poetiki Dostoevskogo, Moscou, 1963, traduction française La Poétique de Dostoïevski, Paris, Seuil, 1970.

Bally Claude, Le langage et la vie, Genève, Droz, [1913] 1965.

Benveniste Émile, Problèmes de linguistique générale, tomes I et II, Paris, Gallimard, 1966 et 1974.

Berrendonner Alain, Éléments de pragmatique linguistique, Paris, Minuit, 1981.

Bres Jacques, La narrativité, Louvain-La-Neuve, Duculot, 1994.

Cadiot Pierre, «Sur le "sens opposé" des mots », Langages n 150, 2003, n 31-47.

Carel Marion et Ducrot Oswald, « Problème du paradoxe dans une sémantique argumentative », Langue Française $\mathrm{n}^{\circ}$ 123, 1999, pp. 6-26.

Carel Marion et Ducrot Oswald, « Mise au point sur la polyphonie », Langue française, $\mathrm{n}^{\circ} 164,2009$, pp. 33-43.

Ducrot Oswald, Dire et ne pas dire, Paris, Minuit [1972] 1980.

Ducrot Oswald, La preuve et le dire, Paris, Mame, 1973.

Ducrot Oswald, Les échelles argumentatives, Paris, Minuit, 1980.

Ducrot Oswald, Le dire et le dit, Paris, Minuit, 1984.

Ducrot Oswald et alii, Les mots du discours, Paris, Minuit, 1980.

Genette Gérard, « Discours du récit », Figures III, Paris, Seuil, 1972.

Genette Gérard, Nouveau discours sur le récit, Paris, Seuil, 1983.

Hagège Claude, « Langue globale, pensée unique », Émission Les grandes questions, Sur France 5, 14.11.2013.

Hjelmslev Louis, Prolégomènes à une théorie du langage, Paris, Minuit, [1943] 1968. 
Maingueneau Dominique, Le Discours littéraire, paratopie et scène d'énonciation, Paris, Armand Colin, 2004.

Nemo François, « Indexicalité, unification contextuelle, et constituant extrinsèque du référent », Langages vol. 37, $\mathrm{n}^{\circ}$ 150, 2003, pp. 88-105.

Nølke Henning, Le regard du locuteur 2. Pour une linguistique des traces énonciatives, Paris, Kimé, 2001a.

Nølke Henning, « La Scalopine 2001, version révisée de la théorie Scandinave de la Polypnonie linguistique ", in Nølke Henning, Polyphonie-linguistique et littéraire, vol. III, 2001b, pp. 43-65.

Nølke Henning, Fløttum Kjersti et Norén Coco, ScaPoLine, La théorie scandinave de la polyphonie linguistique, Paris, Kimé, 2004.

Perrin Laurent, « Aspects de la voix du locuteur à l'intérieur du sens », Cahiers de Praxématique n 49, 2007, pp. 79-102.

Rabatel Alain, Une histoire du point de vue, Metz, Klincksieck, 1997.

Rabatel Alain, « Le point de vue, entre langue et discours, description et interprétation : état de l'art et perspectives ", Cahiers de paxématique ${ }^{\circ}$ 41, 2003, pp. 7-24.

Rabatel Alain, « Analyse énonciative du point de vue, narration et analyse du dicours », Filologia lingüística. portuguesa $\mathrm{n}^{\circ}$ 9, 2007, pp. 345-368.

Rabatel Alain, Homo narrans. Pour une analyse interactionnelle du récit. Tome 1. Les points de vue et la logique de la narration, Limoges, Lambert-Lucas, 2008a.

Rabatel Alain, Homo narrans. Pour une analyse interactionnelle du récit. Tome 2. Dialogisme et polyphonie dans le récit, Limoges, Lambert-Lucas, 2008b.

Rabatel Alain, « Perspective et point de vue », Communications vol. 85, nº 1, 2009, pp. 23-35.

Ricœur Paul, Temps et récit 1, Paris, Seuil, 1983.

Raccah Pierre-Yves, « Lexique et idéologie : les points de vue qui s'expriment avant qu'on ait parlé », in Carel Marion (ed.), Les facettes du dire : Hommage à Oswald Ducrot, Paris, Kimé, 2002, pp. 241-268.

Raccah Pierre-Yves, « Une sémantique du point de vue : de l'intersubjectivité à l'adhésion », in «L'Énonciation identitaire : entre l'individuel et le collectif », dir. par Danielle Forget, Discours social, vol. XXI, 2005a, pp. 205-242.

Raccah Pierre-Yves, « What is an empirical theory of linguistic meaning a theory of? », in Zygmunt Frajzyngier, David Rood and Adam Hodges (eds.), Diversity and Language Theories, Amsterdam, Benjamins, 2005b, pp. 51-80.

Raccah Pierre-Yves, « La pista de los puntos de vista: teoría, descripciones y pruebas en semántica », Letras de Hoje ${ }^{\circ}$ 139, 2005c, pp. 23-50.

Raccah Pierre-Yves (ed.), Signes, langues et cognition, Paris, L'Harmattan, 2005d.

Raccah Pierre-Yves, « Polyphonie et argumentation : des discours à la langue (et retour) », in Simonffy Zsuzsa (ed.), L’un et le multiple, Budapest, TINTA Könyvkiadó, 2006, pp. 120-152.

Raccah Pierre-Yves, « Contraintes linguistiques et compréhension des énoncés : la langue comme outil de manipulation », Entretiens d'orthophonie, 2008, pp. 61-90.

Raccah Pierre-Yves, « Racines lexicales de l'argumentation : la cristallisation des points de vue dans les mots ", Verbum vol. XXXII, nº 1, 2011, pp. 119-141. 
Raccah Pierre-Yves, « Points de vue, idéologie et manipulation », in Raccah Pierre-Yves (dir.), L'inscription des biais socio-cognitifs dans la langue, Limoges, Lambert-Lucas, 2014, pp. 11-34.

Saussure Ferdinand de, Cours de linguistique générale, Paris, Payot, [1916] 1972.

Sperber Dan et Wilson Deirdre, Meaning and Relevance, Cambridge University Press, 2012.

Tordesillas Marta, La argumentación ; 'pourtant' en la dinámica argumentativa de los Essais de Montaigne, Madrid, Édiciones U.C.M, 1992.

Tordesillas Marta, « Esbozo de una teoría dinámica de la lengua en el marco de una semántica argumentativa », Signo y seña $n^{\circ}$ 9, 1998, pp. 349-378.

Tordesillas Marta, «Histoires concessives. Du sens des formes à la forme des sens : le cas de "pourtant" ", in La lingüística francesa en el último milenio, Santiago de Compostela, Arrecife ediciones, 2000, pp. 1047-1061.

Tordesillas Marta, «À propos du signe linguistique : énonciation, argumentation et stéréotype », in El Valor de la [Meta]diversidad, Madrid, Ediciones U.A.M, 2004a, pp. 1930-1950.

Tordesillas Marta, «Los planos del discurso. Fundamentos teóricos para una nueva semántica », Actas I del VIII Simposio Internacional de Comunicación Social, Cuba, Centro de Lingüística Aplicada, 2004b, pp 321-325.

Tordesillas Marta, «La enunciación: fundamentos de lenguaje, principios de lengua, perspectiva docente », in Vera Sant'Anna et Bruno Deusdara, Trajetórias em Enunciaçiao e Discurso, conceitos e práticas, Río de Janeiro, Clara Luz Editora, 2007, pp. 122-144.

Tordesillas Marta, «À propos du signe linguistique : énonciation, argumentation et stéréotype », in Moreno Sandoval, A. (ed.), El valor de la diversidad, UAM, 2008, pp. 1930-1950.

Voloshinov, V. N, МАРКСИЗм И ФИЛосОФИЯ ЯЗЫКА [Le marxisme et la philosophie du langage], Leningrad, Priboj, traduction française, sous le nom de Mikhaïl Bakhtine (V.N. Volochinov), Le marxisme et la philosophie du langage. Essai d'application de la méthode sociologique en linguistique, Paris, Minuit, [1929], 1977.

\section{NOTES}

1. Cet article s'inscrit dans le cadre du projet de recherche financé par le MINECO: FFI2012-38299, Diccionario electrónico de redes discursivas de la lengua francesa a partir de un corpus semánticamente anotado, DERDIS, qui vise à identifier la subjectivité dans la langue moyennant un traitement automatique.

2. De là aussi la problématique qui tourne autour de l'enseignement de la langue de signes ou de la langue «naturelle » aux personnes sourdes par rapport aux imaginaires qu'elles véhiculent du fait que les deux types de langues sont susceptibles de créer des constructions différentes de mondes, d'imaginaires, d'intéractions, etc. Nous n'entrerons pas dans le débat.

3. Hagège réfléchit à la langue globale, la pensée unique, le monde unique, le néoliberalisme, l'intérêt économique, la loi du profit par la loi du néolibéralisme, l'idéologie. Il parle d'une menace actuelle pour l'espèce. Il ajoute que l'homo sapiens s'est développé par différenciation progressive et pas par homogénéisation, pas par uniformité, sans aucune différenciation, ni diversité. On assiste, d'après Hagège (2013), à un cul-de-sac involutif, du fait que l'anglais soit un vecteur unique.

4. Il est à signaler que les fondements théoriques proposés par Ducrot vont devenir plus radicaux au fur et à mesure que les théories se développent dans le temps. 
5. Voir chap. 13.

6. Ducrot, Anscombre, Plantin, Raccah, Bruxelles, Mc Evoy, Zuber, Moeschler, Reboul, etc.

7. Marion Carel, María Marta García Negroni, Sibylle Sauerwein, Silvia Palma, Marta Tordesillas, etc.

8. Tel est le cas par exemple de Carel, qui propose la Théorie des blocs sémantique, et de Tordesillas qui, de son côté, formule une Sémantique argumentative et énonciative, SAE et avec elle une Didactique argumentative et énonciative, DAE ; une Grammaire argumentative et énonciative, GAE ; une Théorie littéraire argumentative et énonciative, TLAE.

9. La prise de conscience en est fondamentale pour la Sciende que l'élan contemporain ne soit pas renversé.

10. Une des actions, par exemple, qui est récemment considérée comme innovatrice en didactique, dans l'enseignement, c'est le travail en groupe ou l'échange d'idées et de perspectives ou prise de positions, processus d'ailleurs reflété dans le Cadre européen commun de référence pour les langues. Ceci dit, ce qui est le plus choquant c'est que, au XXI ${ }^{\mathrm{e}}$ siècle, le travail en groupe et en coopération puisse être considéré comme une nouveauté pour la plupart de la communauté scientifique! L'être humain devrait connaître et, d'ailleurs, connaît bien que les actions en équipe sont beaucoup plus solides que celle qui sont individuelles et, alors, comment se fait-il que l'enseignement, pendant des siècles, se soit développé dans l'individuel! Les hommmes primitifs avaient déjà compris cela et allaient en groupe à la chasse.

11. Cela se produit au XIX ${ }^{\mathrm{e}}$ siècle, quand il s'agit d'étudier la fonction du langage dans le cadre de plusieurs disciplines. Voir Bally ([1965] 1913).

12. Tel est le cas de Benveniste (1966) et (1974).

13. "Quel est l'objet à la fois intégral et concret de la linguistique? La question est particulièrement difficile [...] D'autres sciences opèrent sur des données d'avance et qu'on peut considérer ensuite à différents points de vue; dans notre domaine rien de semblable ", dira Saussure ([1916] $1972: 24)$.

14. Raccah parle de "curiosité », or plusieurs auteurs font appel à cette idée selon laquelle les mots renferment des discours et même sont le résultats de discours. «[...] la Langue comme activité manifestée dans les instances de discours qui sont caractérisées comme telles par des indices propres ", dit Benveniste (1966: 257). L'auteur fait appel à la delocutivité. Elle sera étudiée par Anscombre (1979). Les mots sont la synthèse de discours préalables : «nous ferons par extension l'hypothèse que les mots sont des modules possibles - même les plus utilisés - mais pas les seuls, de véhiculer de façon synthétique les discours de la langue ", formule Tordesillas (2000 : 1051). Voir aussi Tordesillas (2004a).

15. «Si l'on n'efface pas la distinction conceptuelle entre concept et sens, on peut alors voir l'art de la parole comme consistant précisément à construire et proférer des énoncés qui seront interprétés par les interlocuteurs de telle manière que les processus au moyen desquels ils construiront un sens pour ces énoncés les amèneront, au passage, à ces concepts. Ainsi, la possibilité d'accéder à des concepts sans énonciation ne garantit pas un sens sans énonciation mais offre, au contraire, une raison d'être pour certaines énonciations ", indique Raccah (2005a : 215-216).

16. Notamment, Raccah et Tordesillas.

17. Sperber et Wilson en sont la preuve, tel que l'on peut le constater dans leurs recherches les plus récentes : Wilson et Sperber (2012).

18. La plupart des ouvrages liés à la narratologie présentent la théorie de Genette comme le repère scientifique pour la description du récit et cela a duré pendant plus de 20 ans.

19. Rabatel (2009) renvoie à la notion de figure à partir de la conception de plusieurs auteurs : sujet modal de Bally, focalisateur de Bal, foyer de Genette, énonciateur de Ducrot, sujet de conscience de Zribi-Hertz et de Banfield, foyer d'empathisation de Kuno et de Forest, centre de perspective de Lintvelt et de Rabatel, etc. 
20. « Envisager » est à comprendre au sens théorique le plus large qui va, en fonction des cadres théoriques, de la perception au dire.

21. Berrendonner (1981: 52) introduit la notion de "complexe illocutoire", destinée à rassembler, dans une position illocutoire unique, les différentes attitudes du locuteur.

22. Notre travail de thèse doctorale, pour rendre compte des nuances énonciatives clés pour expliquer l'évolution du sens conclusif au sens concessif de «pourtant » du XIV ${ }^{e}$ au XIX ${ }^{e}$ siècles, nous avons dû formuler de nouveaux concepts argumentatifs et de nouvelles figures énonciatives (Tordesillas 1992).

23. Pour une conception énonciative et argumentative du signe, voir Tordesillas (2008).

24. Il est à remarquer la différence entre la proposition de Raccah et celle de Tordesillas. Raccah attribue les relations d'accord, désaccord, identification des énonciateurs face aux points de vue. Tordesillas (2007) formule aussi des liens relativement semblables, mais des locuteurs avec les énonciateurs, alors que les énociateurs convoquent des points de vue positifs, négatifs ou neutres, avec une pojection topique favorable, défavorable, non favorable.

25. Pour aborder le développement des articulateurs et de l'interprétation, Raccah expose les deux modèles topiques et développe la description sémantique du connecteur mais, qui transforme un couple de phrases $<\mathrm{A}, \mathrm{B}>$ en une phrase [A mais $\mathrm{B}]$ ), obtenue, d'après l'auteur, par abstraction à partir des analyses des interprétations possibles en situations des énoncés possibles de la phrase [A mais B], analyses tenant compte des influences des différents points de vue préalables possibles des interprètes sur ces interprétations : la description qui en résulte est donc indépendante des situations et des points de vue, bien qu'elle s'applique à toute situation et tout point de vue. Pour plus de renseignements, consulter Raccah (2014).

26. Le concept de topos, en tant que principe genéral, partagé et graduel, est formulé par Ducrot, dans les années 80 , et précisé par Raccah au long des recherches ultérieures, en spécifiant en 1990 un type de topoi descriptifs et un autre d'heuristiques, ou bien ajoutant par exemple l'idée de catégorie topique ou encore en établissant une sorte d'équation pour sa représentation métalinguistique. Par ailleurs, Raccah fait appel tout aussi au caractère idéologique des points de vue, à des croyances plus ou moins conscientes, puis, d'autre part, l'auteur mentionne des connaissances expertes, des savoirs-faire. Pour pouvoir discriminer entre les deux derniers types de points de vue, il est nécessaire de recourir à des informations extra-linguistiques (statut des inférences, statut cognitif des interlocuteurs, statut social, objectifs, etc...). Ainsi, c'est le statut conféré au texte ou au discours qui permet de décider si les points de vue qu'il exprime relèvent de l'idéologie ou de l'expertise. On peut s'approcher de cette formulation, dans Raccah (ed.) (2005d).

27. Voir Raccah $(2002,2005 b)$ pour des précisions sur ces points.

\section{RÉSUMÉS}

Cette étude comporte une caractérisation de certains concepts essentiels de la linguistique générale, notamment de la sémantique des langues. À partir d'une réflexion sur différents axiomes scientifiques sur la langue, cet article développe des principes de sémantique concernant l'argumentation et l'énonciation dans la langue. Sous une perspective historique de la Science qui rend compte, à chaque fois d'une façon plus radicale, de la subjectivité de / dans la langue à partir d'une conception dynamique de la langue, se développe la conception du point de vue, qui devient primordiale dans les études de sémantique, ainsi que dans plusieurs domaines 
scientifiques qui réfléchissent à la langue. Le point de vue devient d'ailleurs le centre d'une proposition théorique, telle que la Sémantique des points de vue, proposée par Pierre-Yves Raccah. Ladite théorie pose une conception manipulatoire de la langue et montre qu'il est nécessaire d'admettre que les mots ordinaires imposent des contraintes sur les points de vue que les énoncés peuvent évoquer. Elle rend compte de la manière dont les langues imposent à leurs énoncés des biais socio-cognitifs, que les interlocuteurs perçoivent dans les discours qu'ils entendent.

This paper describes some essential concepts of General Linguistics, specially of Languages Semantics. From a reflection concerning different scientific axioms about language, this paper develops principles of Semantics related to argumentation and enunciation in language. Within a historical perspective of science that describes, in a more radical way each time, subjectivity of / in language from a dynamic language conception, we can see how the concept of point of view evolves and becomes a major concept in Semantics studies, as well as in several other scientific domains that reflects on language. Thus, the point of view is the core of Semantics of Points of View, a theoretical proposition promoted by Pierre-Yves Raccah. This theory upholds a manipulative conception of language, shows the need to acknowledge the constraints that ordinary words enforce on the points of view that may be communicated by utterances and describes how languages determine socio-cognitive bias in utterances, which are perceived by interlocutors when they listen to discourses.

\section{INDEX}

Mots-clés : langue, sémantique, subjectivité, énonciation, polyphonie, argumentation, topoi, instructions, contraintes, discours, énoncé, mot-de-discours, mot-de-langue, voix, point de vue. Keywords : Language, Semantics, subjectivity, enunciation, polyphony, constraints, discourse, utterance, part of speech, part of language, voice, point of view.

\section{AUTEUR}

\section{MARTA TORDESILLAS}

Universidad Autónoma de Madrid 